484

\section{CHILDREN'S BLOOD PRESSURE AND PHYSICAL ACTIVITY}

M. Calvo Terrades ${ }^{1}$, J.C. Sevilla Moya ${ }^{2,3,4}$, S. Espuña Díez ${ }^{2}$, C. Javierre Garcés ${ }^{5}$, R. Jiménez González 6

${ }^{1}$ Albera Salut, ABS Peralada. Girona Institute for Biomedical Research, Peralada Gerona, ${ }^{2}$ Fundació Salut Empordà, Figueres, ${ }^{3}$ Girona Institute for Biomedical Research, Girona,

${ }^{4}$ Hospital Universitario La Paz, Madrid, ${ }^{5}$ Physiology Department, Medicine School, Barcelona University, ${ }^{6}$ University Hospital Joan de Déu (Esplugas de Llobregat), Department of Paediatrics, School of Medicine, University of Barcelona, Barcelona, Spain

Primary prevention of cardiovascular diseases and promotion of active lifestyle should start early. Blood pressure (BP) in childhood correlates with BP in adulthood. The knowledge about children's physical activity influence on BP is limited.

Aims: To analyse the relation between physical activity (PhyA) and time of "screen-activities" (ST) performed by children and BP.

Methods: 428 children were enrolled, provided parent's consent. Measurements: BP: systolic (syst) and diastolic (diast) Z-score, pulse pressure $(\mathrm{PP})$, mean pressure (PM). Activity information was obtained from:

1) a seven-day questionnaire,

2) the K13Plus PhyA and sedentary behaviour scale (K13 PhyS).

Based on Z-score BP values participants were classified in: High-BP $(\mathrm{HBP}) \geq 1.03$ (n: syst=46, diast=100), Normal-BP (NBP) $<1.03$ (n: syst=382, diast=328).

\section{Results:}

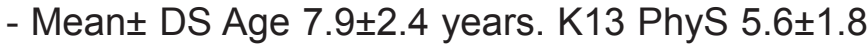
points. Screen-time $65.3 \pm 43.4$ minutes/day.

PhysA intensity (minutes/day): low 35.5 \pm 32.2 , moderate $49,9 \pm 43.6$, vigorous $29.2 \pm 21.6$, moderate+vigorous $78.7 \pm 49.2$

- Correlation's analysis (significant results, ( ${ }^{*}$ almost) Pearson C.) : Moderate+vigorous PhyA: ZScoreBPsyst $(-0,163)$, ZScoreBPdias $(-0,116)$, PP $\left(-0,102^{*}\right)$, PM $(-0,16)$.
Moderate PhyA: ZScoreBPsyst $\left(-0,102^{*}\right)$, PP $(-0,115), \operatorname{PM}(-0,135)$.

Vigorous PhyA: ZScoreBPsyst $(-0,16)$

K13 PhyS: ZScoreBPsyst $(-0,107)$

Screen-time: $\operatorname{PP}(0,12)$

- Comparison of means showed: less mean time/ day of moderate, and moderate+vigorous PhyA in HBPsyst (HBP $38.3 \pm 28,7 ; 63.7 \pm 39,4$. NBP $50,8 \pm 49,1 ; 82,7 \pm 80,0)$.

\section{Conclusions:}

- Children studied just reach current PhyA and "screen-time" recommendations.

- PhyA have an effect on children's BPvalues.

- Mean time/day of moderate+vigorous PhyA seems to be the best preventive factor; inversely "screentime"/day.

- PhyA and BP at childhood relationship must be considered as protective cardiovascular risk factors.

485

\section{EVALUATION OF ORAL HYGIENE HABITS IN A PORTUGUESE PRIMARY SCHOOL CHILDREN POPULATION}

H. Rodrigues ${ }^{1}$, A. Lamas ${ }^{2}$, C. Ribeiro ${ }^{1}$, A. Torres ${ }^{1}$, J. Rios ${ }^{1}$, A. Araújo ${ }^{1}$, I. Maciel ${ }^{1}$

${ }^{1}$ Pediatrics, Unidade Local de Saúde do Alto Minho, EPE, ${ }^{2}$ Clínica Dentária A Lamas, Viana do Castelo, Portugal

Background and aims: Good oral hygiene habits should be implemented very soon in children's development. It is important to quantify the improvements and the dental plaque index can be an easy mean to do it.

Methods: Prospective study, with the application of an anonymous questionnaire to the parents of 194 primary school children of Viana do Castelo, Portugal. During the study period we taught the children how to brush their teeth and we made an evaluation of their dental plaque prior and after it.

Results: The children's age was comprised between $5-9$ years and $52 \%$ were females. Only $20 \%$ of the parents started to brush their children's teeth before 1 year. Ninety-eight percent brush their teeth 
alone, only once a day in $26 \%$ of the cases. Only $11 \%$ use dental floss, after 6 years in $37 \%$ of the cases. Eighty-two percent have already gone to a dentist, only $49 \%$ for prevention. Thirty-four percent of the parents say their children don't eat cariogenic foods. Seventy-three percent eat sweets frequently and $72 \%$ drink refrigerants. Only $44 \%$ of the parents said their children's oral hygiene is good and only $14 \%$ knew that caries is an infectious disease. The mean index of dental plaque before and after the teaching was $89 \%$ and $52 \%$, respectively (reduction of $42 \%)$.

Conclusions: We observed a significant reduction in dental plaque with our taught and this let us conclude that this should be an issue to invest not only in the consultations, but also in schools and community settings.

\section{6}

\section{AGE AT CRYPTORCHIDISM DIAGNOSIS AND ORCHIOPEXY: A POPULATION-BASED STUDY OF 502,815 DANISH BOYS BORN FROM 1995 TO 2009}

\begin{abstract}
M.S. Jensen ${ }^{1,2}$, L.H. Olsen ${ }^{3}$, A.M. Thulstrup ${ }^{2}$, J.P. Bonde ${ }^{4}$, J. Olsen ${ }^{5}$, T.B. Henriksen ${ }^{1}$

${ }^{1}$ Perinatal Epidemiology Research Unit, Department of Pediatrics, Aarhus University Hospital, Skejby, ${ }^{2}$ Department of Occupational Medicine, Aarhus University Hospital, ${ }^{3}$ Pediatric Urology and Research Unit, Department of Urology, Aarhus University Hospital, Skejby, Aarhus, ${ }^{4}$ Department of Occupational and Environmental Medicine, Bispebjerg Hospital, University of Copenhagen, Copenhagen, ${ }^{5}$ The Institute of Public Health, University of Aarhus, Aarhus, Denmark
\end{abstract}

Background and aims: Early treatment of cryptorchidism may be necessary to preserve fertility and international guidelines recommend that congenital cryptorchidism be treated with orchiopexy before one year of age. Acquired cryptorchidism should be treated at presentation. The adherence to guidelines during recent years is not known, and the aim of this study is to present data on age at cryptorchidism diagnosis and orchiopexy in recent Danish birth cohorts.

Methods: A population of 502,815 Danish boys born alive from January 1, 1995 to October 21, 2009 was identified using The Danish Civil Registration System. Five birth cohorts were defined, 1995-1997, 1998-
2000, 2001-2003, 2004-2006 and 2007-2009. The boys were followed in the Danish National Registry of Patients for a diagnosis of cryptorchidism and for an orchiopexy procedure. Data was analyzed using the Kaplan-Meier estimator and Cox regression models.

Results: During follow-up, 9,479 boys were diagnosed with cryptorchidism and 5,076 of these underwent orchiopexy. The mean age (years) at diagnosis among boys followed at least 5.8 years was: 1995-1997 cohort, 3.30 (95 \% confidence interval: 3.23; 3.37); 1998-2000 cohort, 3.08 (3.01; $3.15) ; 2001-2003$ cohort, 2.79 (2.73; 2.86). The corresponding mean age (years) at orchiopexy was: 1995-1997 cohort, 3.75 (3.65; 3.85); 1998-2000 cohort, 3.54 (3.45; 3.63); 2001-2003 cohort, 3.18 (3.10; 3.26). Restriction of the analyses to 427,650 term (gestational age $\geq 37$ weeks) singletons did not substantially change the results.

Conclusions: In the more recent birth cohorts of 1995 to 2006 we found a shift towards younger age at cryptorchidism diagnosis and orchiopexy.

487

\section{VIRTUAL PATIENTS: AN EFFECTIVE EDUCATIONAL INTERVENTION TO IMPROVE SENIOR HOUSE OFFICERS' EDUCATION AND TRAINING IN THE MANAGEMENT OF CHILD ABUSE?}

\author{
M. McEvoy ${ }^{1}$, B. Butler ${ }^{2}$, A. Nicholson ${ }^{3}$, \\ G. MacCarrick ${ }^{4}$
}

${ }^{1}$ Paediatrics, The Children's University Hospital, ${ }^{2}$ RCSI Media Department, ${ }^{3}$ The Children's University Hospital, Temple Street, ${ }^{4} \mathrm{RCSI}$ Department of Medical Education, RCSI, Dublin, Ireland

As a junior doctor is often the only doctor to assess children presenting with an injury or illness to the A\&E department, it is crucial that they are skilled in the identification of possible child abuse or neglect.

Aim: To evaluate an interactive computer based Virtual Patient (VP) (using videos to simulate real life clinical scenarios) developed to teach paediatric Senior House Officers about the management of suspected child abuse.

Methods: The VP was used to facilitate a child abuse case discussion for Paediatric Basic Specialist Trainees in Ireland. A questionnaire was developed to determine SHOs' perception of the value of the 